Case Report

J Exp Clin Med

2021; 38(2): 204-207

doi: $10.52142 /$ omujecm.38.2.27

\title{
Two different regenerative injections in patients diagnosed with secondary hip osteoarthritis: A report of two cases
}

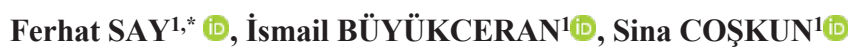

${ }^{1}$ Department of Orthopaedics and Traumatology, Faculty of Medicine, Ondokuz Mayıs University, Samsun, Turkey

Received: $14.04 .2020 \quad \bullet \quad$ Accepted/Published Online: $16.12 .2021 \quad \bullet \quad$ Final Version: 14.03 .2021

\begin{abstract}
Non-surgical treatment options are limited in hip osteoarthritis secondary to developmental dysplasia of the hip (DDH). Treatment of Autologous Conditioned Serum (ACS) is based on the high level of anti-inflammatory cytokine production of leukocytes in peripheral blood. It is an effective treatment option which contains the cytokines and the growth factors. Platelet Rich Plasma (PRP) is a form of treatment that stimulates natural healing steps through growth factors in platelets. In this case report we aimed to present the results of ACS and PRP injection in two patients who developed secondary coxarthrosis after DDH surgery. At the 1-year follow-up examination, patient's functional scores improved and requirement for non-steroid anti-inflammatory drugs had significantly reduced. Regenerative injections either autologous conditioned serum or platelet rich plasma treatments are safe and effective treatments and can be recommended particularly for cases of secondary hip osteoarthritis associated with DDH.
\end{abstract}

Keywords: Autologous conditioned serum, developmental dysplasia of the hip, platelet rich plasma

\section{Introduction}

Primary osteoarthritis (OA) is most often seen in the hip joint after the knee. One of the most common reasons for secondary OA in the hip joint is the developmental dysplasia of the hip (DDH). Cases of DDH, which are not effectively treated during childhood, experience pain in the hip and may lead to secondary hip OA in adulthood. Medications in current use for pharmacological treatment of OA include analgesics, nonsteroid anti-inflammatory drugs (NSAID), opioids, corticosteroids, visco-supplementation, glucosamine and chondroitin sulfate. Treatment alternatives are being researched which will reduce cartilage degeneration and modify the disease to provide a symptomatic effect for OA. Growth factors, matrix-metalloproteinase inhibitors, cytokines, nitric oxide and gene therapies are treatment choices, which are being researched in this area (Fajardo and Di Cesare, 2005).

The catabolic effect of interleukine-1 (IL-1) in the OA process is known (Goldring, 2001; Tetlow et al., 2001). IL-1 receptor antagonist (IL-1Ra), which is a surface receptor antagonist of IL-1, is present in the serum as a cytokine (Arend et al., 1998). IL-1Ra is bound to IL-1 receptors but cannot pull the trigger for any intracellular response. Autologous conditioned serum (ACS) treatment is based on a high level of anti-inflammatory cytokine production (especially IL-1Ra) by leukocytes in the peripheral blood (Evans, 2005; Wehling et al., 2007; Auw Yang et al., 2008; Baltzer et al., 2009; Baltzer et al., 2013; Frizziero et al., 2013;).

Platelet Rich Plasma (PRP) is a treatment form stimulating natural healing steps through growth factors contained in the platelets. PRP applied to the wound area accelerates the physiological healing process, provides support for the connection of cells, reduces pain and has an anti-inflammatory and anti-bacterial effect (Sanchez et al., 2009). Obtaining PRP growth factor is a simple, cheap and easy way (Alsousou et al., 2009). As it is autogenous in origin, easy to prepare and has an excellent reliability profile, it has opened the door to new treatment (Lopez-Vidriero et al., 2010). Studies in literature have reported good results with the use of either ACS or PRP in degenerative joint pathologies (Auw Yang et al., 2008; Baltzer et al., 2009; Sanchez et al., 2012; Baltzer et al., 2013; Dallari et al., 2016; Say et al., 2013; Tassara et al., 2018). In this case report, the treatment results are presented of two patients diagnosed with secondary hip OA who were treated with ACS and the other with PRP. The patients were informed that data concerning cases would be submitted for publication, and they provided consent. 


\section{Case report}

\subsection{Case 1}

A twenty-three-year-old man presented to the orthopedics department with the complaint of pain in the left hip. It was learned from the history that the pain in the hip had been ongoing for six years and in the last year, the patient had been taking NSAID every day and he had restricted movement. When the patient was 1.5 years old, he had been operated on for DDH and there was nothing else remarkable in the history. In the physical examination, there was no limb length discrepancy and restriction in joint movement was determined in the left hip. Goniometer measurements determined left hip flexion $100^{\circ}$, extension $15^{\circ}$, abduction $30^{\circ}$, adduction $15^{\circ}$, internal rotation $20^{\circ}$ and external rotation $40^{\circ}$. On direct radiographs, deformation in the left hip joint, narrowness in the joint space in the acetabulum and subchondral sclerosis and osteophytic projections were detected (Fig. 1). On magnetic resonance imaging (MRI), a subchondral cystic change was determined in the left acetabulum in addition to degenerative changes (Fig. 2). It was planned to administer an ACS injection to the left hip joint. The patient had a baseline Harris Hip Score of 60 and Visual Analog Scale (VAS) of 8. Approximately $60 \mathrm{cc}$ of peripheral venous blood was withdrawn from the patient into an injector and covered with chrome sulphate medical spheres. After 24 hours incubation at $37^{\circ} \mathrm{C}$, it was centrifuged for 10 mins. After separation of the serum, it was stored at $-18^{\circ} \mathrm{C}$. Under operating room conditions and fluoroscopy guidance, a total of 3 doses of ACS injection were administered at 1-week intervals (Fig. 3). At the 6-month follow-up examination, the requirement for NSAID had significantly reduced. The angles of movement of the left hip were measured as flexion $110^{\circ}$, extension $20^{\circ}$, abduction $35^{\circ}$, adduction $20^{\circ}$, internal rotation $20^{\circ}$ and external rotation $40^{\circ}$. The Harris Hip Score was 81 and VAS was determined as 5. At the 1-year follow-up examination, there was a negligible need for NSAID; Harris Hip Score was determined as 90 and VAS as 2.

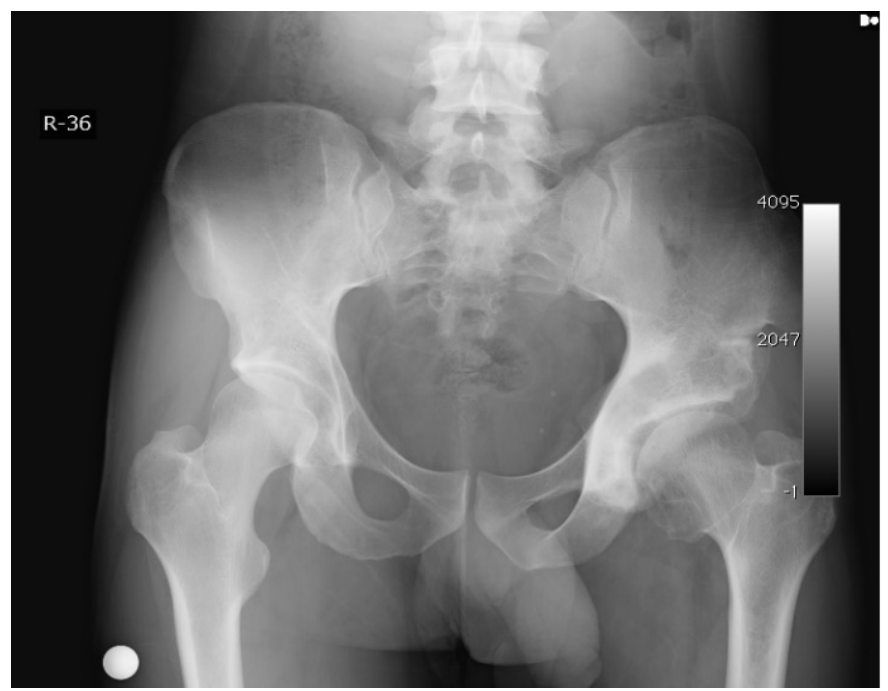

Fig. 1. Pelvic X-Ray of the first case showing deformation in the left hip joint, narrowness in the joint space in the acetabulum and subchondral sclerosis and osteophytic projections

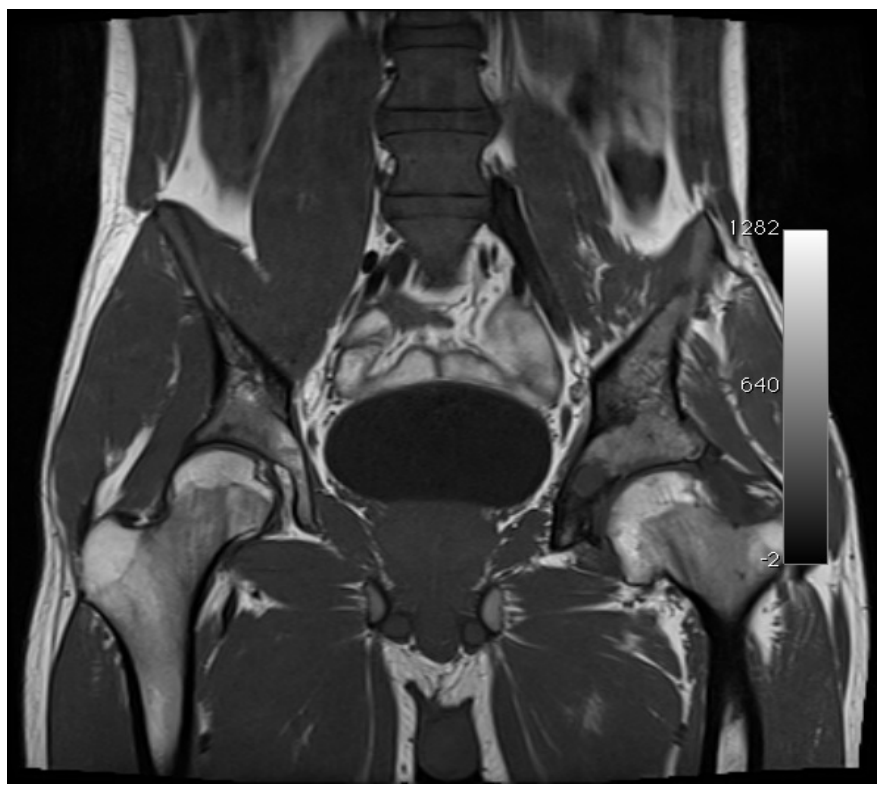

Fig. 2. T1-weighted coronal plane pelvic Magnetic Resonance Imaging of the case 1

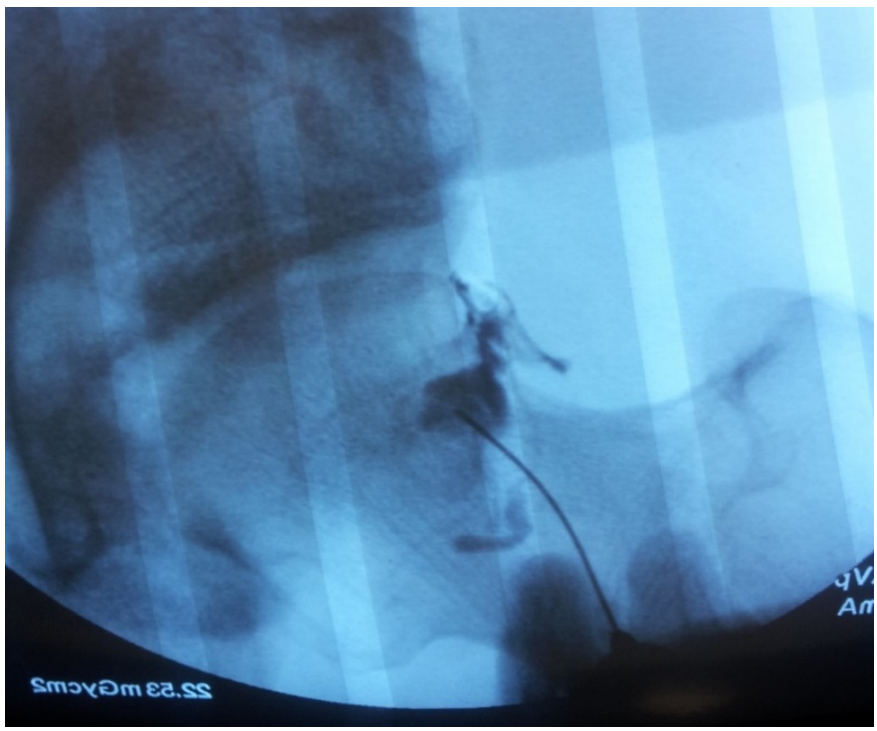

Fig. 3. Fluoroscopy guided hip ACS injection after confirming with radioopaque

\subsection{Case 2}

A twenty-eight-year-old woman presented to the orthopedics department with the complaint of pain in the left hip. It was learned from the history that the pain in the hip had been ongoing for one year and the patient had been taking NSAID twice a week. When the patient was 13 years old, she had been operated on for DDH and there was nothing else remarkable in the history. In the physical examination, there was no limb length discrepancy and restriction in joint movement was determined in the left hip. Goniometer measurements determined left hip flexion $110^{\circ}$, extension $25^{\circ}$, abduction $40^{\circ}$, adduction $25^{\circ}$, internal rotation $25^{\circ}$ and external rotation $40^{\circ}$. On direct radiographs, deformation in the left hip joint, narrowness in the joint space in the acetabulum and subchondral sclerosis and osteophytic projections were detected (Fig. 4). It was planned to administer a PRP injection to the left hip joint. The patient had a baseline Harris Hip Score of 80 and VAS as 8 . 
In the preparation of PRP Anitua's method was used. A total of $30 \mathrm{cc}$ peripheral blood was taken from antecubital region of the patient into tubes containing 3.2\% sodium citrate. The tubes were centrifuges at $1800 \mathrm{rpm}$ for eight minutes at room temperature. Under operating room conditions and fluoroscopy guidance a single dose of $3.5 \mathrm{ml}$ activated PRP containing $5.5 \%$ calcium chloride $\left(\mathrm{Cl}_{2} \mathrm{Ca}\right)\left(50 \mu \mathrm{Cl}_{2} \mathrm{Ca}\right.$ in $1 \mathrm{ml}$ PRP) was administered to the left hip.

At the 1-year follow-up examination, there was a negligible need for NSAID; Harris Hip Score was determined as 90 and VAS as 6. There was no difference in hip movement measurements except $10^{\circ}$ increases in internal rotation.

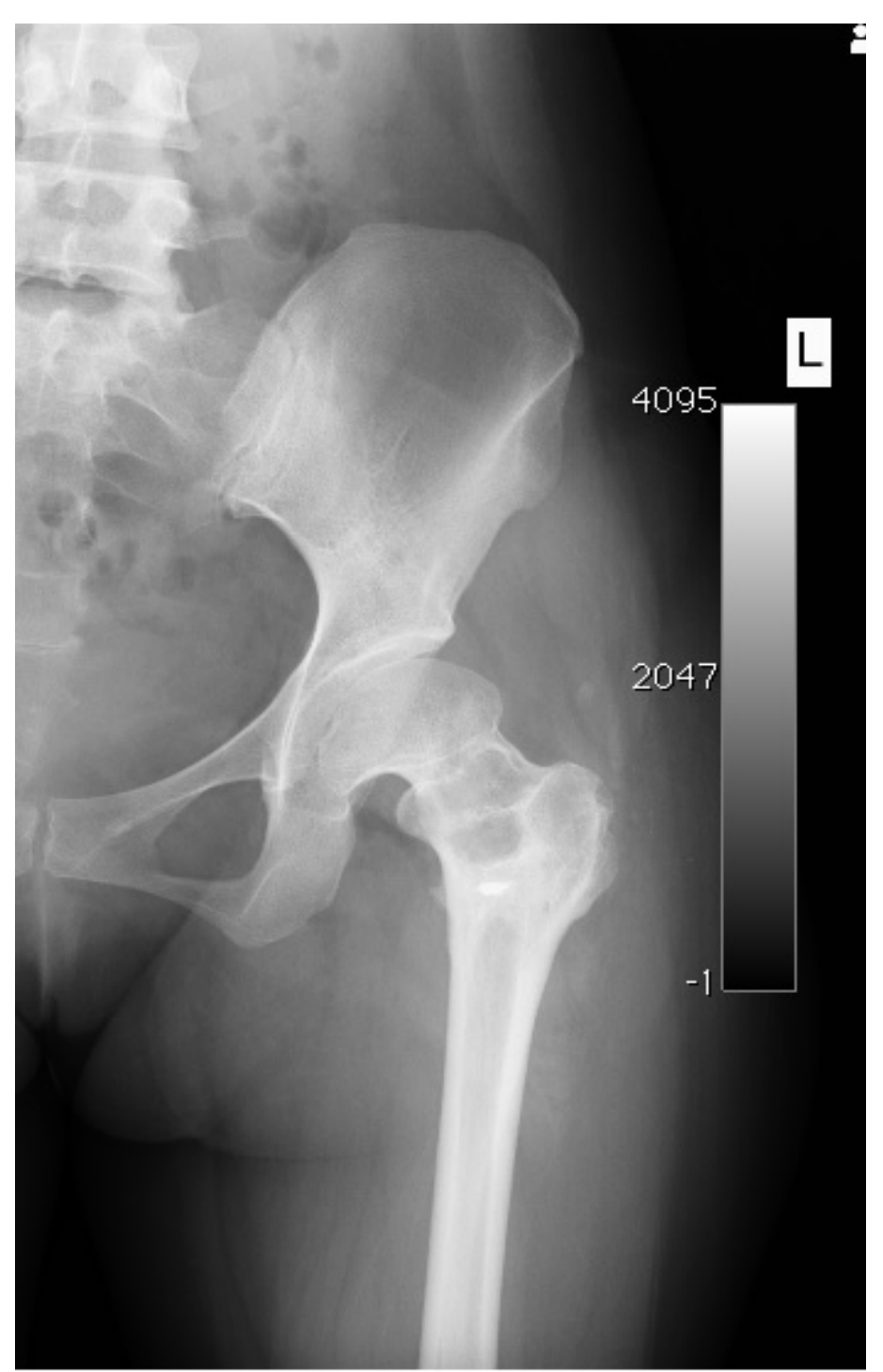

Fig. 4. Left hip X-Ray of the second case

\section{Discussion}

In patients with secondary OA associated with $\mathrm{DDH}$, treatment planning is important as they are of a younger age with a longer life expectancy. Surgical choices for the treatment of these patients include arthroscopy, osteotomy and total hip arthroplasty (Kosuge et al., 2013). The disadvantages of surgical treatment are that it is invasive, with high costs, lengthy hospital stay and high complication rates. NSAIDs are the most commonly used current non-surgical treatment.
However, the side effects of chronic use on the gastrointestinal, renal and cardiovascular systems restrict NSAID treatment (Harirforoosh et al., 2013). Corticosteroid and viscosupplementation applied to the hip joint have the negative aspects of the effects being short-lived, symptomatic effects being shown and the potential side effects of steroids (Abate et al., 2008; Kruse, 2008). There is a need for new, non-invasive treatments for hip OA which will modify the disease.

In recent years, successful results in hip and knee OA treatment have been reported from the use of PRP containing cytokines and growth factors obtained from autologous blood (Sanchez et al., 2012; Say et al., 2013; Dallari et al., 2016). Also, treatments directed at the inhibition of IL-1, which is responsible for the inflammatory changes in OA, have attracted attention in recent years. In a 2-year follow-up of early-stage knee OA patients treated with ACS, pain was reported to have decreased and function increased (Baltzer et al., 2009). In another study, a decrease in pain was reported in an 18-month follow-up period of hip OA patients (Baltzer et al., 2013). The complaints of the current cases on presentation were of negative effects on quality of life because the restrictions in movement were causing difficulties in daily functions. Longterm use of NSAID requires kidney function testing at intervals and additional medication for gastrointestinal complaints. Following the application of the ACS and PRP treatments, the need for NSAID was significantly reduced and with the improvement in joint range of movement, the difficulties in daily activities were resolved. After treatments, both patients resumed an active working life.

During incubation period of ACS, the contact of blood cells with the medical spheres increases the IL-1Ra level 140-fold (Frizziero et al., 2013). Together with IL-1Ra, ACS also contains anti-inflammatory cytokines such as interleukine-4 and interleukine-10, insulin-like growth factor (IGF-1) and growth factors such as platelet-derived growth factor (PDGF) (Wehling et al., 2007; Frizziero et al., 2013). It is thought that the effect of ACS is not contained in a single component, but that it occurs through the synergistic effect of all the factors (Wehling et al., 2007). Besides the reduction of pain, ACS treatment also has the clinical protective and regenerative effect on cartilage of growth factors such as PDGF and IGF-1 (Evans, 2005).

Growth factors such as platelet-derived growth factor, transforming growth factor, vascular endothelial growth factor and insulin like growth factor and proteins such as fibrin, fibronectin, vitronectin, and thrombospondin, which are found in PRP, play a role in many stages of tissue healing. The growth factors activate some of the cells which have a function in tissue healing and thus provide soft tissue healing and bone regeneration (Alsousou et al., 2009). The mechanism of the effect of PRP on the degenerative knee joint can be listed as inflammation regulation by means of the growth factors and proteins contained in it, correction of angiogenesis, anabolic 
and cartilage protective effects, cell differentiation and synovial cell modulation. However, as there is not a single mechanism of the effects of PRP on joint pathologies, it is more complex than previously thought (Andia et al., 2012).

As ACS and PRP treatments are obtained from autologous blood, there is no risk of immune reaction or disease transfer. There are no studies in literature warning of hyperplasia, carcinogenesis or tumor growth of PRP (Sanchez et al., 2012). Septic arthritis reported in 1 patient was associated with the injection procedure rather than the content of ACS (Auw Yang et al., 2008). No side effects were determined in the cases reported here. In the 1-year follow-up of the current cases diagnosed with hip OA secondary to DDH and treated with ACS and PRP, pain reduced and functionality increased.

In conclusion, regenerative injections either ACS or PRP treatments can be recommended particularly for cases of secondary hip OA associated with DDH. However, there is a need for further prospective, randomized, placebo-controlled, multi-centre studies to enable a clearer understanding of these findings and the effects of ACS and PRP.

\section{Conflict of Interest}

This case report has no conflict of interest

\section{Acknowledgements}

This case report has no source of funding.

\section{References}

1. Abate, M., Pelotti, P., De Amicis, D., Di Iorio, A., Galletti, S, Salini,V. 2008. Viscosupplementation with hyaluronic acid in hip osteoarthritis (a review). Ups. J. Med. Sci. 113, 261-277.

2. Alsousou, J., Thompson, M., Hulley, P., Noble, A., Willett, K. 2009. The biology of platelet-rich plasma and its application in trauma and orthopaedic surgery: a review of the literature. J. Bone Joint Surg. Br. 91, 987-996.

3. Andia, I., Sanchez, M., Maffulli, N. 2012. Joint pathology and platelet-rich plasma therapies. Expert. Opin. Biol. Ther. 12, 7-22.

4. Arend, W.P., Malyak, M., Guthridge, C.J., Gabay, C. 1998. Interleukin-1 receptor antagonist: Role in biology. Annu. Rev. Immunol. 16, 27-55.

5. Auw Yang, K.G., Raijmakers, N.J., van Arkel, E.R., Caron, J.J., Rijk, P.C., Willems, W.J., Zijl, J.A., Verbout, A.J., Dhert, W.J., Saris, D.B. 2008. Autologous interleukin-1 receptor antagonist improves function and symptoms in osteoarthritis when compared to placebo in a prospective randomized controlled trial. Osteoarthritis Cartilage. 16, 498-505.

6. Baltzer, A.W., Moser, C., Jansen, S.A., Krauspe, R. 2009. Autologous conditioned serum (Orthokine) is an effective treatment for knee osteoarthritis. Osteoarthritis Cartilage. 17, 152-60.

7. Baltzer, A.W., Ostapczuk, M.S., Stosch, D., Seidel, F., Granrath, M. 2013. A new treatment for hip osteoarthritis: Clinical evidence for the efficacy of autologous conditioned serum. Orthop. Rev. 5, 5964.

8. Dallari, D., Stagni, C., Rani, N., Sabbioni, G., Pelotti, P., Torricelli, P., Tschon, M., Giavaresi, G. 2016. Ultrasound-Guided Injection of Platelet-Rich Plasma and Hyaluronic Acid, Separately and in Combination, for Hip Osteoarthritis: A Randomized Controlled Study. Am. J. Sports. Med. 44, 664-671.

9. Evans, C.H. 2005. Novel biological approaches to the intra-articular treatment of osteoarthritis. BioDrugs. 19, 355-362.

10. Fajardo, M., Di Cesare, P.E. 2005. Disease-modifying therapies for osteoarthritis : current status. Drugs Aging. 22, 141-61.

11. Frizziero, A., Giannotti, E., Oliva, F., Masiero, S., Maffulli, N. 2013. Autologous conditioned serum for the treatment of osteoarthritis and other possible applications in musculoskeletal disorders. Br. Med. Bull. 105, 169-84.

12. Goldring, M.B. 2001. Anticytokine therapy for osteoarthritis. Expert Opin. Biol. Ther. 1, 817-29.

13. Harirforoosh, S., Asghar, W., Jamali, F. 2013. Adverse effects of nonsteroidal antiinflammatory drugs: An update of gastrointestinal, cardiovascular and renal complications. J. Pharm. Pharm. Sci. 16, 821-847.

14. Kosuge, D., Yamada, N., Azegami, S., Achan, P., Ramachandran, M. 2013. Management of developmental dysplasia of the hip in young adults: current concepts. Bone Joint J. 95-B, 732-737.

15. Kruse, D.W. 2008. Intraarticular cortisone injection for osteoarthritis of the hip. Is it effective? Is it safe? Curr. Rev. Musculoskelet Med. 1, 227-233.

16. Lopez-Vidriero, E., Goulding, K.A., Simon, D.A., Sanchez, M., Johnson, D.H. 2010. The use of platelet-rich plasma in arthroscopy and sports medicine: optimizing the healing environment. Arthroscopy. 26, 269-278.

17. Sánchez, M., Anitua, E., Orive, G., Mujika, I., Andia, I. 2009. Platelet-rich therapies in the treatment of orthopaedic sport injuries. Sports Med. 39, 345-54.

18. Sánchez, M., Guadilla, J., Fiz, N., Andia, I. 2012. Ultrasound-guided platelet-rich plasma injections for the treatment of osteoarthritis of the hip. Rheumatology (Oxford). 51, 144-50.

19. Say, F., Gürler, D., Yener, K., Bülbül, M., Malkoc, M. 2013. Platelet-rich plasma injection is more effective than hyaluronic acid in the treatment of knee osteoarthritis. Acta. Chir. Orthop. Traumatol. Cech. 80, 278-283.

20. Tassara, M., De Ponti, A., Barzizza, L., Zambelli, M., Parisi, C., Milani, R., Santoleri, L. 2018. Autologous conditioned serum (ACS) for intra-articular treatment in Osteoarthritis: Retrospective report of 28 cases. Transfus. Apher. Sci. 57, 573-577.

21. Tetlow, L.C., Adlam, D.J., Woolley, D.E. 2001. Matrix metalloproteinase and proinflammatory cytokine production by chondrocytes of human osteoarthritic cartilage: associations with degenerative changes. Arthritis Rheum. 44, 585-594.

22. Wehling, P., Moser, C., Frisbie, D., Mcllwraith, C.W., Kawcak, C.E., Krauspe, R., Reinecke, J.A. 2007. Autologous conditioned serum in the treatment of orthopedic diseases: the orthokine therapy. BioDrugs. 21, 323-332. 